\title{
Membrane Binding and Insertion of a pHLIP Peptide Studied by All-Atom Molecular Dynamics Simulations
}

\author{
Yonghua Deng ${ }^{1}$, Zhenyu Qian ${ }^{1}$, Yin Luo ${ }^{1}$, Yun Zhang ${ }^{1}$, Yuguang Mu ${ }^{2}$ and Guanghong Wei ${ }^{1, *}$ \\ 1 State Key Laboratory of Surface Physics, Key Laboratory for Computational Physical Sciences \\ (Ministry of Education), and Department of Physics, Fudan University, 220 Handan Road, \\ Shanghai 200433, China; E-Mails: 072019007@fudan.edu.cn (Y.D.); \\ 09110190008@fudan.edu.cn (Z.Q.); 09110190006@fudan.edu.cn (Y.L.); \\ 0529009@fudan.edu.cn (Y.Z.) \\ 2 School of Biological Sciences, Nanyang Technological University, 60 Nanyang Drive, \\ Singapore 637551, Singapore; E-Mail: ygmu@ntu.edu.sg \\ * Author to whom correspondence should be addressed; E-Mail: ghwei@fudan.edu.cn; \\ Tel.: +86-21-5566-5231; Fax: +86-21-6510-4949.
}

Received: 31 May 2013; in revised form: 24 June 2013 / Accepted: 25 June 2013 /

Published: 12 July 2013

\begin{abstract}
Recent experiments in function mechanism study reported that a $\mathrm{pH}$ low-insertion peptide (pHLIP) can insert into a zwitterionic palmitoyloleoylphosphatidylcholine (POPC) lipid bilayer at acidic $\mathrm{pH}$ while binding to the bilayer surface at basic $\mathrm{pH}$. However, the atomic details of the $\mathrm{pH}$-dependent interaction of pHLIP with a POPC bilayer are not well understood. In this study, we investigate the detailed interactions of pHLIP with a POPC bilayer at acidic and basic $\mathrm{pH}$ conditions as those used in function mechanism study, using all-atom molecular dynamics (MD) simulations. Simulations have been performed by employing the initial configurations, where pHLIP is placed in aqueous solution, parallel to bilayer surface (system $\mathrm{S}$ ), partially-inserted (system P), or fully-inserted (system F) in POPC bilayers. On the basis of multiple 200-ns MD simulations, we found (1) pHLIP in system S can spontaneously insert into a POPC bilayer at acidic $\mathrm{pH}$, while binding to the membrane surface at basic $\mathrm{pH}$; (2) pHLIP in system $\mathrm{P}$ can insert deep into a POPC bilayer at acidic $\mathrm{pH}$, while it has a tendency to exit, and stays at bilayer surface at basic pH; (3) pHLIP in system F keeps in an $\alpha$-helical structure at acidic $\mathrm{pH}$ while partially unfolding at basic $\mathrm{pH}$. This study provides at atomic-level the $\mathrm{pH}$-induced insertion of $\mathrm{pHLIP}$ into POPC bilayer.
\end{abstract}


Keywords: $\mathrm{pH}$ low-insertion peptide (pHLIP); zwitterionic POPC; $\mathrm{pH}$-triggered bilayer insertion; membrane binding; all-atom molecular dynamics simulations

\section{Introduction}

The $\mathrm{pH}$ low-insertion peptides (pHLIPs) have received significant attention in recent years due to their ability to target acidic tissues and selectively translocate polar, cell-impermeable molecules across cell membranes [1,2]. At $\mathrm{pH}$ values above seven, $\mathrm{pHLIP}$ in aqueous solution partitions to the surface of a lipid bilayer without inserting. Under acidic $\mathrm{pH}$ conditions, pHLIP inserts into a lipid bilayer and forms a transmembrane helix [1-5]. Due to its small size, pHLIP serves as a model peptide for studying membrane protein folding and bilayer insertion. Its folding energetics across a palmitoyloleoylphosphatidylcholine (POPC) lipid bilayer has been studied recently by fluorescence spectroscopy and isothermal titration calorimetry [4]. During the insertion process, pHLIP can translocate cargo molecules attached to its $C$-terminus via a disulfide, and release them in the cytoplasm of the cell $[1,5,6]$. Mutation study showed that Asp residues in the $C$-terminal region of pHLIP are critical for solubility and $\mathrm{pH}$-dependent membrane insertion of the peptide $[7,8]$. Unlike other membrane active peptides [9], pHLIP helices cause minimal disturbance to phospholipid bilayers: Namely, they do not induce membrane leakage $[1,6,10]$. Owing to this exceptional property, many technologies have been developed for targeting, imaging, and drug molecule delivery using pHLIP [7,8,11-14].

A 35-aa peptide, with the sequence AEQNPIYWAR ${ }^{10}$ YADWLFTTPL $^{20}$ LLLDLALLVD $^{30}$ ADEGT [3], is one of the $\mathrm{pH}$ low-insertion peptides. This pHLIP is a water-soluble peptide derived from the transmembrane helix $\mathrm{C}$ of the integral membrane proteins Bacteriorhodopsin [15] and displays a pH-triggered membrane insertion behavior [3]. In function mechanism study of the $\mathrm{pH}$-dependent membrane insertion of pHLIP, the reported $\mathrm{pH}$ values for bilayer insertion and exit of this pHLIP peptide are 4.0 and 8.0, respectively [3]. In spite of well-performed experimental study, the atomic details of $\mathrm{pH}$-induced bilayer insertion of this pHLIP peptide are not well understood.

Molecular dynamics (MD) simulations can provide detailed information on peptide-membrane interactions. A number of MD simulation studies have investigated the lipid interactions and the energetics of membrane insertion of different peptides/proteins [16-21]. The potential of mean force (PMF) for the transfer of pHLIP across lipid bilayers has been investigated by a coarse-grained MD study [16], which provides theoretical insights into the thermodynamics of pHLIP in membrane environment. However, the pH-dependent pHLIP-membrane interaction at atomic level is yet to be determined.

The goal of this study is to investigate the detailed interaction of pHLIP with a zwitterionic POPC lipid bilayer at different $\mathrm{pH}$ conditions. We have performed multiple, independent 200-ns MD simulations at acidic and basic $\mathrm{pH}$ starting from three different initial configurations where the initial structure of pHLIP is an $\alpha$-helix. The reason of choosing the $\alpha$-helical structure as the starting state of our MD simulations is discussed in detail in the Experimental Section. Noting, the pHLIP responds to the $\mathrm{pH}$ change from neutral to mildly acidic $\mathrm{pH}$ in biomedical experiments $[7,8]$. In the mechanism 
study of pHLIP in solution when POPC vesicles were employed instead of live cells [3], the used pHs were $\mathrm{pH} 4$ and $\mathrm{pH}$ 8. Following this experimental study [3], such acidic and basic $\mathrm{pHs}$ (pH 4 and $\mathrm{pH} 8$ ) were chosen for our simulation study [3]. In the initial configurations, pHLIP is placed parallel to bilayer surface (system S), partially-inserted (system P), or fully-inserted (system F) in the POPC bilayer. The membrane binding process and insertion behavior of pHLIP, as well as the pHLIP-POPC interactions at acidic and basic $\mathrm{pH}$ are to be investigated. This study provides molecular insight into the mechanism of $\mathrm{pH}$-triggered membrane insertion for $\mathrm{pHLIP}$ peptides.

\section{Results and Discussion}

To mimic the experimental acidic $\mathrm{pH}$ ( $\mathrm{pH} 4.0$ ) condition [3], the side chains of Asp, Glu, Arg, and the $\mathrm{N}$-terminus are protonated (Asp0, Glu0, $\mathrm{Arg}^{+}, \mathrm{NH}_{3}{ }^{+}$). In order to examine the role of protonation of Asp/Glu residues in the transmembrane and $C$-terminal region on the bilayer insertion of pHLIP, and exclude the influence of $C$-terminus it is taken as deprotonated (COO-) (with the same protonation state as that at basic $\mathrm{pH}$ ), while $\mathrm{COO}$ - and $\mathrm{COOH}$ coexist around $\mathrm{pH}$ 4.0. In a basic $\mathrm{pH}(\mathrm{pH}$ 8.0) environment, the side chains of Asp and Glu and the $N$ - and $C$-termini are deprotonated (Asp-, Glu-, $\mathrm{NH}_{2}$ and COO-), and the side chain of Arg is protonated $\left(\mathrm{Arg}^{+}\right)$. The pHLIP carries one net positive charge at this acidic $\mathrm{pH}$ and six net negative charges at this basic $\mathrm{pH}$. It should be noted that, in reality, the $\mathrm{pK}$ of each Asp/Glu shifts with the surrounding environment (the protonation state of Asp/Glu will change accordingly). As the calculation of $\mathrm{pK}$ is very computational costly, we neglect the $\mathrm{pK}$ shift in our MD simulations, as done recently by other groups [18,19,22-24].

We have carried out multiple, independent 200-ns MD simulations starting from the three different initial states S, P, F described in Experimental Section. Spontaneous bilayer insertion of pHLIP at acidic $\mathrm{pH}$ is relatively rare in our performed MD simulations at $315 \mathrm{~K}$. We have launched five MD runs for system $\mathrm{S}$ (acidic) (data not shown), while insertion event is only observed in one MD trajectory. MD runs of the $\mathrm{P}$ and $\mathrm{F}$ systems are used to further confirm the insertion property observed in $\mathrm{S}$ (acidic). Here we present only the results of six representative MD runs, named as $\mathrm{S}$ (acidic), $\mathrm{S}$ (basic), $\mathrm{P}$ (acidic), $\mathrm{P}$ (basic), F (acidic), and $\mathrm{F}$ (basic). We first investigate the $\mathrm{pH}$-dependent insertion behavior of pHLIP in system S, where pHLIP was initially placed in aqueous solution with its backbone parallel to the POPC bilayer surface and the center-of-mass (COM) of the pHLIP backbone is $2.2 \mathrm{~nm}$ away from the membrane surface (see the snapshot at $t=0 \mathrm{~ns}$ in Figure 1a,b).

\subsection{In Aqueous Solution, pHLIP Can Spontaneously Insert into POPC Bilayer at Acidic pH, while} Binds to the Membrane Surface without Inserting at Basic pH

Figure 1 presents the $\mathrm{pH}$-dependent $\mathrm{pHLIP-POPC}$ interaction in the two MD trajectories of $\mathrm{S}$ (acidic) and $\mathrm{S}$ (basic). The snapshots generated at five different time points in the two MD runs are displayed in Figure 1a,b. The time evolution of the insertion depth of pHLIP in POPC lipid bilayer is shown in Figure 1c. The bilayer insertion depth is probed by two parameters: One is the z-position of the COM of peptide backbone and the other the z-position of the most deeply inserted atom of residue Leu28. In acidic $\mathrm{pH}$ environment, the pHLIP moves toward the hydrophilic headgroup of the POPC bilayer and is adsorbed to bilayer surface within the first $5 \mathrm{~ns}$, with its $C$-terminal residues binding to the headgroup region of the bilayer. This is followed by a spontaneous insertion of the 
$C$-terminal residues into the hydrophobic tail region of the bilayer and at $t=25 \mathrm{~ns}$, the $C$-terminal residue Leu28 is located $0.5 \mathrm{~nm}$ below the phosphorous atoms $(z=2.05 \mathrm{~nm}$ ) (see red curve in Figure 1c). It takes some time for the peptide to across the headgroup region. A full insertion of the pHLIP inside POPC bilayer is observed at $t=50 \mathrm{~ns}$, with a z-position of $1.5 \mathrm{~nm}$ for backbone COM and $0.55 \mathrm{~nm}$ for the most deeply inserted atom of Leu28. Thereafter the peptide continues to move toward the hydrophobic center and at $t=100 \mathrm{~ns}$, the $z$-position of the deepest atom of Leu28 reaches to $0.5 \mathrm{~nm}$, although the backbone COM is still located at $z=1.5 \mathrm{~nm}$. Interestingly, although the pHLIP is initially placed parallel to the membrane surface, the $C$-terminal residues firstly binds to the bilayer surface and directs the whole chain to insert into the bilayer. This is in accordance with the experiment results that the pHLIP insertion across a membrane is unidirectional with the $C$-terminal going first to insert [1,7].

Figure 1. Detailed analysis of the two MD trajectories for system $\mathrm{S}$ at acidic and basic pHs. Snapshots at five different time points in the MD runs of (a) $S$ (acidic) and (b) $\mathrm{S}$ (basic). The peptide is shown in ribbon. The phosphorous atoms of the lipids are shown in tan spheres to display the bilayer surface. The other atoms of POPC lipid molecules and water molecules are not shown for clarity. The bilayer center is set at $z=0$;

(c) The $z$-position of the center-of-mass (COM) of pHLIP backbone atoms and the z-position of the most-deeply embedded atom in residue Leu28 as a function of time. The two dashed black lines at $z=2.05 \mathrm{~nm}$ and $z=0 \mathrm{~nm}$ correspond to the bilayer surface (the headgroup phosphorus atoms) of the upper leaflet and the bilayer center, respectively.

(a) S(acidic)
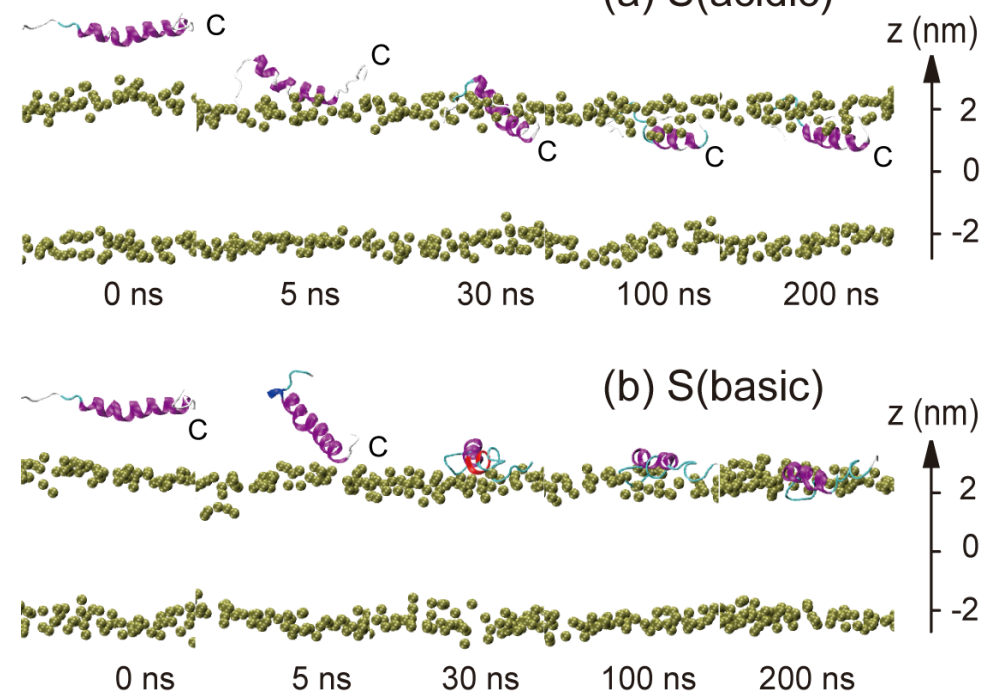

(C)

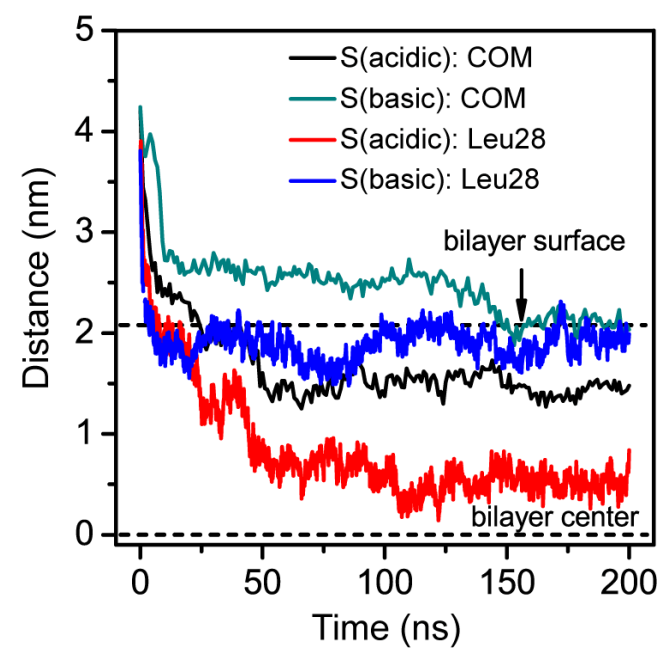

Similar MD simulations studied on other peptides such as alamethicin [25], magainin [26], protegrin-1 [27], Buforin II [22,24], and WALP [23] have been reported to investigate peptide-lipid interactions. Insertion of WALP peptides (ace-AWW-(LA) $5_{5}-\mathrm{WWA}$-ame and ace-GWW-(LA) ${ }_{8} \mathrm{~L}-\mathrm{WWA}$-ame) into DPPC bilayer was observed in high-temperature $\left(>80{ }^{\circ} \mathrm{C}\right) \mathrm{MD}$ simulations [23]. Interestingly, spontaneous insertion of Buforin II and Transportan 10 peptides into POPC bilayer was also observed in recent MD simulations at $300 \mathrm{~K}[19,22,24]$. 
At basic $\mathrm{pH}$, the peptide is also quickly adsorbed to the bilayer surface within the first 5 ns (see Figure 1b), while its backbone $\operatorname{COM}(z=2.7 \mathrm{~nm})$ is still in the aqueous solution (see Figure 1c). At $t=30 \mathrm{~ns}$ the peptide stays at the water-bilayer interface with its backbone parallel to bilayer surface (see the snapshot in Figure 1b) and a z-position of backbone COM of $2.7 \mathrm{~nm}$. At $t=150 \mathrm{~ns}$, the backbone COM and the most deeply inserted atom of Leu28 both reach to $z=2.05 \mathrm{~nm}$ (the location of phosphorous atom in the POPC headgroup). The peptide remains there during the remainder of the 200-ns MD simulations. This finding is in good agreement with fluorescence experiments showing that the pHLIP binds to the surface of POPC lipid bilayers at $\mathrm{pH} 8.0$ [3].

To examine the conformational change during the bilayer binding and insertion process of pHLIP, we have plotted in Figure S1 the DSSP profile of pHLIP. At acidic $\mathrm{pH}$, the helix unfolds from the water-exposed $N$-terminal region, during the process of the $C$-terminal residues of pHLIP across the headgroup region. After the peptide fully inserts into the bilayer (after $60 \mathrm{~ns}$ ), some of the unfolded $N$-terminal residues (residues 14-19) start to refold and a helix spanning residue 13-27 is observed at $125 \mathrm{~ns}$. This helical structure maintains during the remainder of the 200-ns MD simulation. At basic $\mathrm{pH}$, the helix unfolds from the $C$-terminal region (residues 24-28) during the bilayer binding process, which is likely due to the strong electrostatic interactions of the negatively charged D24, D30, D32, and E33 residues with the positively charged choline group in the headgroup region of POPC. However, refolding event is not observed within the 200-ns MD trajectory.

\subsection{When Partially Pre-Inserted in POPC Bilayer, pHLIP Can Insert Deep into the Bilayer at Acidic $\mathrm{pH}$ while Moves towards the Bilayer Surface at Basic $\mathrm{pH}$}

To further examine the $\mathrm{pH}$-dependent bilayer insertion properties of $\mathrm{pHLIP}$, we have launched two 200-ns MD runs for the partial-insertion system (system P) at acidic and basic pH solution conditions. The two MD runs are labeled as $\mathrm{P}$ (acidic) and $\mathrm{P}$ (basic). Figure 2 shows the snapshots taken at five different time points and the time evolution of bilayer insertion depth of pHLIP. The bilayer insertion depth is described by two parameters: one is the $z$-position of the peptide backbone COM and the other the $z$-position of the most deeply inserted atom of residue Leu28. In the initial state $(t=0 \mathrm{~ns})$, the z-position is $3.0 \mathrm{~nm}$ for peptide COM and $1.5 \mathrm{~nm}$ for Leu28 (see Figure 2c). It can be seen from Figure 2a,c) that at acidic $\mathrm{pH}$ condition, the pHLIP moves towards the bilayer center. The $z$-positions of peptide COM and residue Leu28 both decrease with simulation time, reaching to respectively $z=1.5 \mathrm{~nm}$ and $0.2 \mathrm{~nm}$ at $t=100 \mathrm{~ns}$. These two parameters remain respectively around the two values in the left period of MD runs and the peptide stays inside the bilayer. This insertion depth is very similar to the one observed in the MD run of S (acidic). In contrast, at basic $\mathrm{pH}$ the pre-inserted $C$-terminal residues move towards the hydrophilic headgroup region of the upper leaflet and have the tendency to exit from the hydrophobic region of bilayer (see Figure 2b,c). The $z$-position of residue Leu28 is $1.0 \mathrm{~nm}$ at $t=200 \mathrm{~ns}$, while it is $0.6 \mathrm{~nm}$ at $t=0 \mathrm{~ns}$, indicative of a tendency of moving to the bilayer surface. The peptide COM is located at $z=2.0 \mathrm{~nm}$ (the $z$-position of bilayer surface) during the last $50 \mathrm{~ns}$. Interestingly, although we place the pHLIP perpendicular to the plane of POPC bilayer in the initial state, the peptide appears to move towards an in-pane orientation and stays near the surface of POPC bilayer. These results provide further supports on the finding observed in the two MD runs of $\mathrm{S}$ (acidic) and $\mathrm{S}$ (basic) given in Figure 1: pHLIP have a tendency to insert into the bilayer at acidic $\mathrm{pH}$ 
while binds to the bilayer surface at basic $\mathrm{pH}$. It is noted that the surface-bound pHLIP is in a partially folded state.

The conformational change of pHLIP during the membrane entry and exit process is also probed by plotting the DSSP profile in Figure S2. At acidic $\mathrm{pH}$, most of the helical region remains with a kink at residues W14 and L15 during the bilayer insertion process. In contrast, at basic $\mathrm{pH}$ condition, the helix unfolds partially during the bilayer exit process. In the final conformation, the initial helix spanning residue $14-18$ is lost, which is likely due to the strong electrostatic interactions of residue D13 and D24 with POPC headgroups (see below for the electrostatic interaction energy given in Figure 6). The conformational deformation of pHLIP helix observed here and that in S (basic) system is probably correlated with the strong electrostatic interactions with lipid headgroups. Interestingly, in a recent MD study on membrane entry of buforin II peptide, it was reported that the extent of structural deformation appeared directly related to the formation of increased lipid interactions [24].

Figure 2. Detailed analysis of the two MD trajectories for system $P$ at acidic and basic pHs. Snapshots at five different time points in the MD runs of (a) P (acidic) and (b) $\mathrm{P}$ (basic). The peptide is shown in ribbon. The phosphorous atoms of the lipids are shown in tan spheres to display the bilayer surface. The other atoms of POPC lipid molecules and water molecules are not shown for clarity. The bilayer center is set at $z=0$; (c) The $z$-position of the center-of-mass (COM) of pHLIP backbone atoms and the $z$-position of the most deeply inserted atom in residue Leu28 as a function of time. The two dashed black lines at $z=2.05 \mathrm{~nm}$ and $z=0 \mathrm{~nm}$ corresponds to the bilayer surface (the headgroup phosphorus atoms) of the upper leaflet and the bilayer center, respectively.
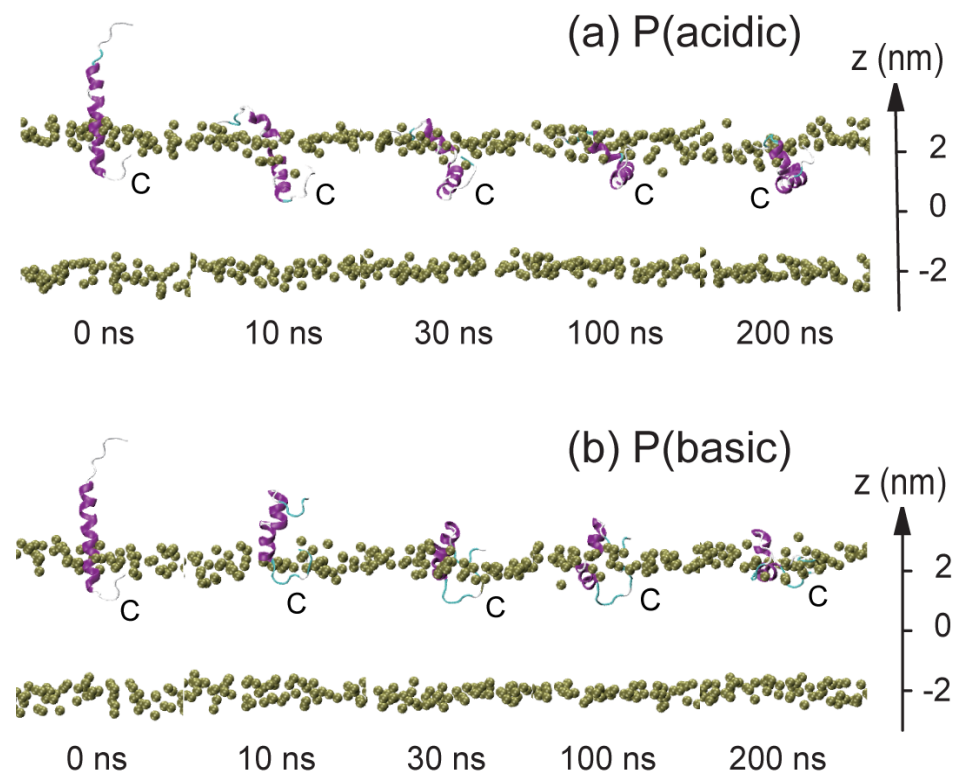

(c)

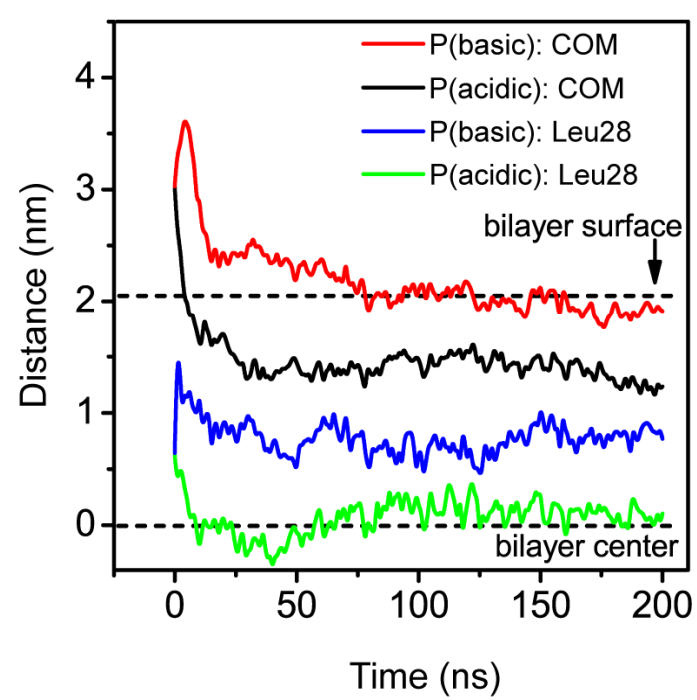

In order to compare the bilayer insertion depth of pHLIP in the four different MD runs, starting from $\mathrm{S}$ and $\mathrm{P}$ configurations, we plot the z-position of the most deeply inserted atom of each residue in Figure 3. It can be seen from Figure 3a,b that starting from two states ( $\mathrm{S}$ and $\mathrm{P}$ ) with different starting positions and orientations of the peptides relative to the bilayer surface, MD runs $\mathrm{S}$ (acidic) and $\mathrm{P}$ (acidic) at acidic $\mathrm{pH}$ both lead to a bilayer-insertion state of the peptide, while MD runs $\mathrm{S}$ (basic) and 
$\mathrm{P}$ (basic) at basic $\mathrm{pH}$ result in a surface-bound state of the pHLIP peptide. Interestingly, we observe that the most hydrophobic region spanning residues W14-V29 are buried deep in the POPC bilayer, with residue L28 being the most deeply bilayer-inserted residue. This is also the reason that we use residue L28 to monitor the insertion depth of the peptide in Figures 1 and 3.

Figure 3. The $z$-position of the most deeply inserted peptide atom of each residue in POPC bilayer. The results are from four different MD runs: (a) $\mathrm{S}$ (acidic) and $\mathrm{S}$ (basic); (b) $\mathrm{P}$ (acidic) and $\mathrm{P}$ (basic). For each MD run, the $z$-position is an average of the last $50 \mathrm{~ns}$.

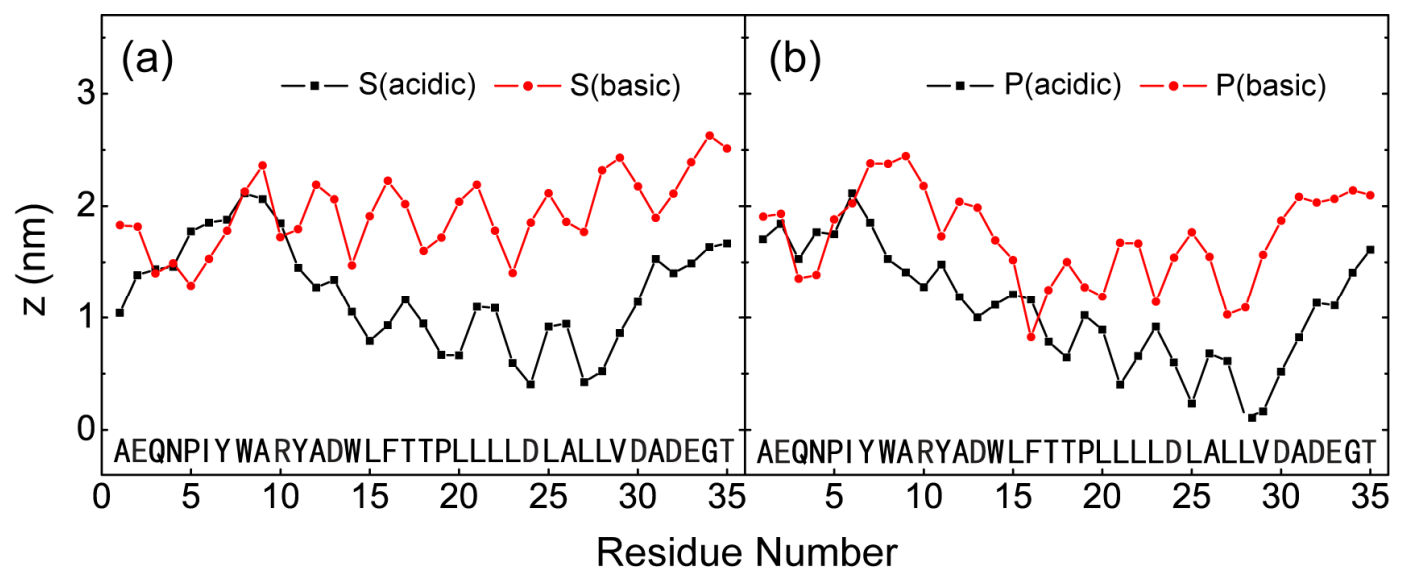

\subsection{When Fully Pre-Inserted inside POPC Bilayer, pHLIP Keeps Intact $\alpha$-Helical Structure at Acidic pH while the Helix Unfolds at Basic $p H$}

In order to probe the stability and the conformation change of the pHLIP inside the POPC bilayer, we have performed MD simulations with pHLIP fully-inserted in the bilayer at both acidic and basic pH. Figure 4 shows the snapshots taken at five different time points and the time evolution of secondary structure profile of the pHLIP. The initial state of pHLIP in the two MD runs is a trans-bilayer $\alpha$-helix. During the full period of 200-ns MD runs of F (acidic), the initial $\alpha$-helix keeps intact. However, it unfolds in the MD run of $\mathrm{F}$ (basic) and loses $30 \%$ of its initial helical structure at $t=10 \mathrm{~ns}$. Helix unfolding mainly initiates from the $C$-terminus. With the increase of simulation time, more helical structure is lost and only $40 \%$ of initial $\alpha$-helix remains at $t=200 \mathrm{~ns}$. It is expected that the $\alpha$-helix will unfold completely if the simulation time is long enough. The helix unfolding implies that $\alpha$-helical structure is not favorable for membrane-bound $\mathrm{pHLIP}$ at basic $\mathrm{pH}$. This is consistent with the experimental results that the pHLIP in the membrane unfolds, from helix to coil, before the exit when triggered by acidity change of $\mathrm{pH}$ from 4 to 8 [3]. However, the spontaneous exit was not observed in our $200 \mathrm{~ns}$ MD run, likely due to the $200 \mathrm{~ns}$ being too short to observe the spontaneous exit of pHLIP (the estimated exit time is 65 milliseconds [3]). 
Figure 4. Detailed analysis of the two MD trajectories (F (acidic) and F (basic)) for full-insertion system at acidic and basic pHs. Snapshots at five different time points in the MD runs of (a) F (acidic) and (b) F (basic). The peptide is shown in ribbon. The phosphorous atoms of the lipids are shown in tan spheres to display the bilayer surface. The other atoms of POPC lipid molecules and water molecules are not shown for clarity. The bilayer center is set at $z=0$. The secondary structure profiles of the pHLIP in the MD runs of (c) F (acidic) and (d) F (basic).

(a)

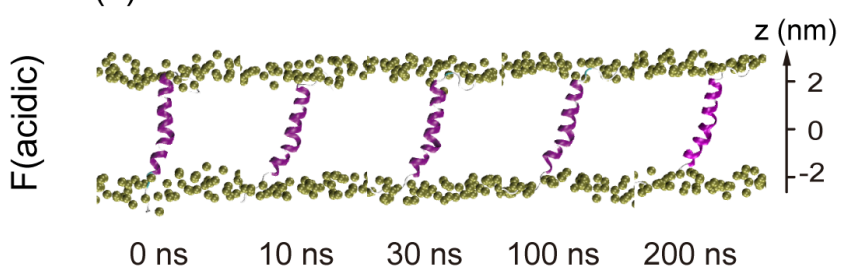

(b)

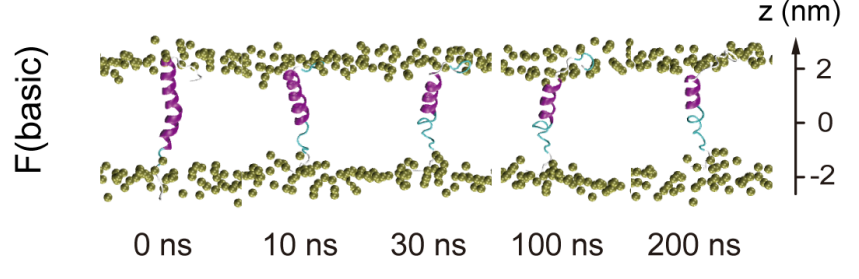

(c)

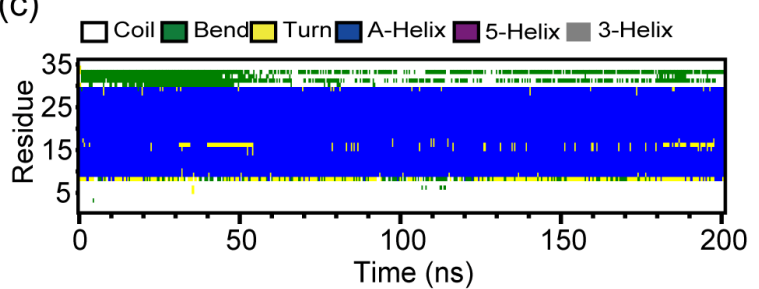

(d)

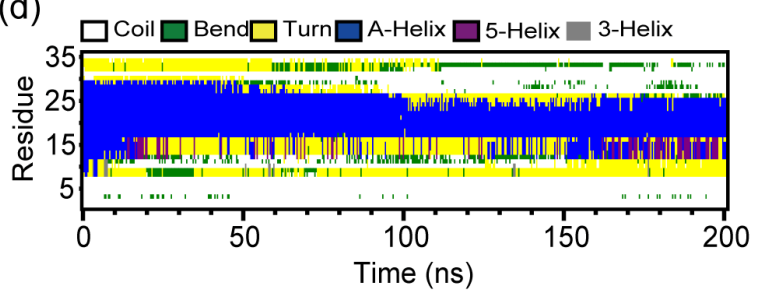

\subsection{The Protonation of Transmembrane Residues Asp and the C-Terminal Residues Asp/Glu}

Facilitates Their Bilayer Entry Due to the Increased Hydrophobicity of pHLIP at Acidic $p H$, while the Strong Electrostatic Interaction between the Deprotonated Asp/Glu Residues and Lipid Headgroups Hinders the Bilayer Insertion at Basic $\mathrm{pH}$

To identify the critical residues and the important physical interactions for $\mathrm{pH}$-dependent pHLIP-POPC interaction, we have first calculated the time evolution of the minimum distance from bilayer center $(z=0)$ for each of the seven polar residues (E2, R10, D13, D24, D30, D32, and E33) in the four MD runs of S (acidic), S (basic), P (acidic), and P (basic). The results are given in Figure 5. It can be seen from Figure 5 that for $\mathrm{S}$ system, at acidic $\mathrm{pH}$ (Figure 5a), pHLIP binds to POPC bilayer surface within the first $25 \mathrm{~ns}$. The transmembrane residue D24 penetrates into the lipid tail region at $\sim 30$ ns. Bilayer insertion of the $C$-terminal residues D30 and D32 is observed at $t=50 \mathrm{~ns}$. This is followed by the membrane entry of residues D13, E33, and E2. During the last $50 \mathrm{ns,}$, all of the Asp and Glu residues stay in the hydrophobic tail region of POPC bilayer, while the positively charge R10 still binds to the hydrophilic headgroup region, which is likely due to strong attractive electrostatic interaction between the positively charged R10 and the negatively charged phosphate group in the headgroups. However, at basic $\mathrm{pH}$ (Figure 5b), all of the Asp and Glu residues bind to the headgroup region of POPC bilayer, which is likely due to the strong attractive electrostatic interaction between the negatively charged E2, D13, D24, D30, and D32 and the positively charged choline groups. When pHLIP partially pre-inserted in the POPC bilayer (P system), at acidic $\mathrm{pH}$ (Figure 5c), transmembrane residue D24 and the $C$-terminal residue D30 insert deep into POPC bilayer, resulting in membrane entry of R10 and D13. During the last $50 \mathrm{ns,}$, all of the Asp and Glu residues are located in the 
hydrophobic tail region of POPC bilayer, with residue D24 and D30 being buried most deeply. However, at basic $\mathrm{pH}$ (Figure 5d), residues D30(-) and D32(-) move to the lipid hydriphilic headgroup region from the hydrophobic tail region. During the last $50 \mathrm{~ns}$, all of the Asp and Glu residues bind to the headgroup region of POPC bilayer.

Figure 5. Time evolution of the minimum distance from bilayer center $(z=0)$ for each of the seven charged residues (E2, R10, D13, D24, D30, D32, and E33) in the four MD runs of S (acidic), S (basic), P (acidic), and P (basic).
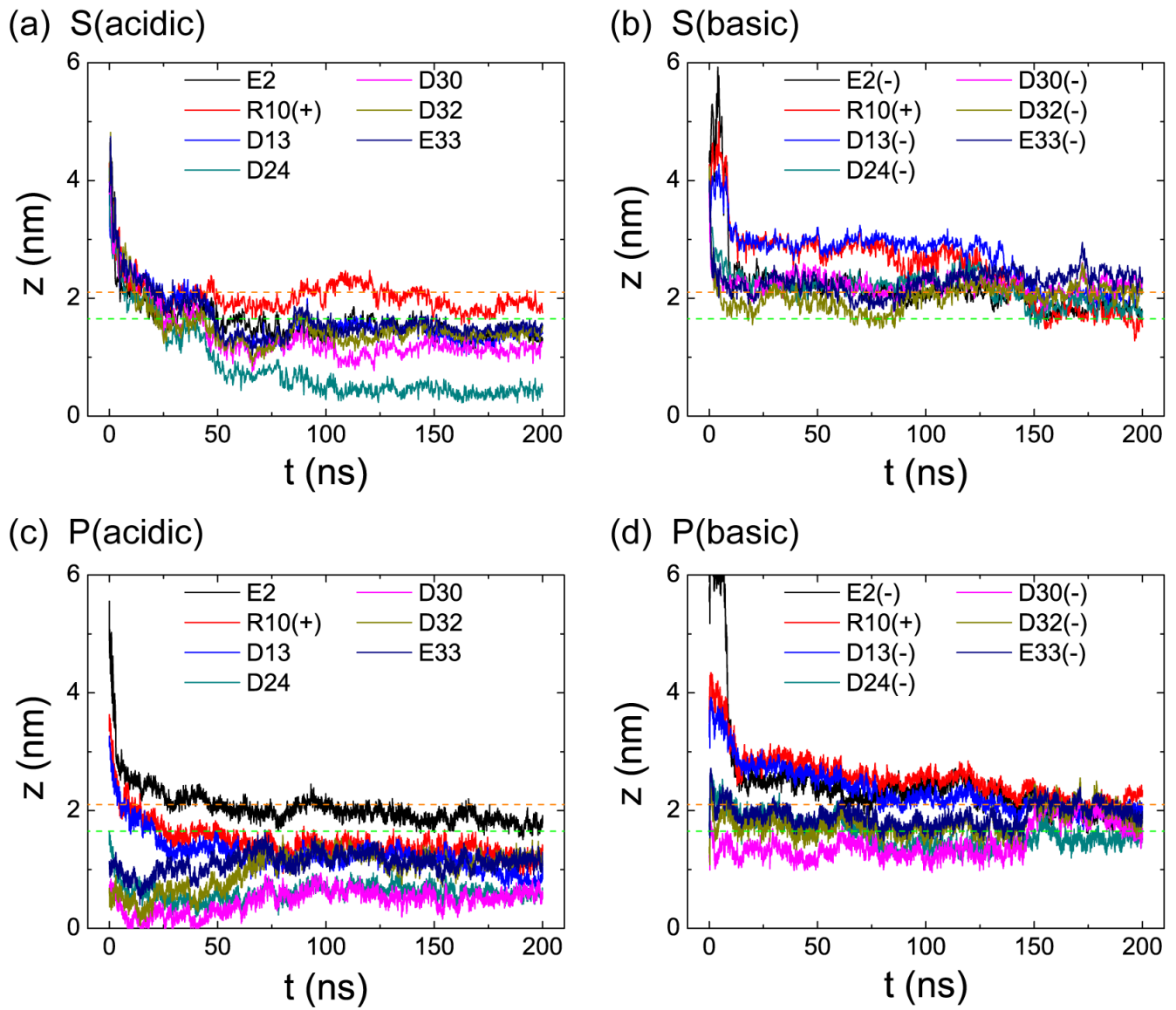

We then plot in Figure 6 the interaction energy between each amino acid residue and POPC lipid bilayer (per lipid) for the six different MD runs starting from the three different initial states. For each MD run, the interaction energy is averaged over the last $50 \mathrm{~ns}$ and decomposed into electrostatic and van der Waals $(v d W)$ components. To compare the $v d W$ interaction of each residue with POPG lipids at acidic and basic $\mathrm{pH}$ conditions, we present in the inset the $v d W$ interaction energy using a different scale. It appears that the insertion depth is not directly correlated with the total $v d W$ interaction energy between each residue and lipid molecule (tail and headgroup). Similar $v d W$ interaction energies are seen in the six different systems. It can be seen from Figure 6 that the interaction energies between charged residues and POPC lipid are much stronger with respect to uncharged residues, indicating that electrostatic interactions play important role on the $\mathrm{pH}$-dependent $\mathrm{pHLIP-POPC}$ interaction. The importance of electrostatic interactions between charged residues and lipid headgroups on the interaction of amyloid peptides with zwitterionic/charged lipid bilayers has also been reported in recent MD studies [28-31]. By comparing the peptide-lipid interaction energy in the left three panels with 
those in the right three panels in Figure 6, we see that the $C$-terminal polar residues Asp30, Asp32, and Glu33 display much stronger electrostatic interactions with POPC lipids at basic $\mathrm{pH}$, which may prevent pHLIP from inserting into the lipid bilayer. The pHLIP has a net charge of -6 at basic $\mathrm{pH}$ and +1 at acidic $\mathrm{pH}$. The peptide at basic $\mathrm{pH}$ is more hydrophilic, energetically more favorable to bind to hydrophilic headgroups of POPC bilayer, whereas the pHLIP at acidic $\mathrm{pH}$ is more hydrophobic, energetically more favorable to stay inside the hydrophobic tail region of POPC bilayer. This view is supported by the time-average potential energy calculation for the whole system (consisting of pHLIP, lipid bilayer, water molecules, and counterions) over the last $50 \mathrm{~ns}$. Given the fact that the simulation timescales are too short to estimate the entropic contributions accurately, our analysis is only qualitative and indicative. The total potential energies of $\mathrm{S}$ (acidic), $\mathrm{P}$ (acidic), and $\mathrm{F}$ (acidic) system are $-5590,-5818$, and $-5835 \mathrm{~kJ} / \mathrm{mol}$, respectively, with the potential energy of $\mathrm{F}$ (acidic) system being the lowest $(-5835 \mathrm{~kJ} / \mathrm{mol})$. The total potential energies of S (basic), P (basic), and F (basic) are -8784 , -9166 , and $-8475 \mathrm{~kJ} / \mathrm{mol}$, respectively, with the potential energy of $\mathrm{F}$ (basic) system being the highest $(-8475 \mathrm{~kJ} / \mathrm{mol})$.

Figure 6. The time-averaged interaction energy between each individual residue of pHLIP and POPC lipids (per lipid) in the six different MD runs: (a) S (acidic); (b) S (basic); (c) $\mathrm{P}$ (acidic); (d) $\mathrm{P}$ (basic); (e) $\mathrm{F}$ (acidic); (f) $\mathrm{F}$ (basic). The interaction energy is averaged over the last $50 \mathrm{~ns}$ of each MD run. The residue-based interaction energy is decomposed into the electrostatic and van der Waals $(v d W)$ terms.

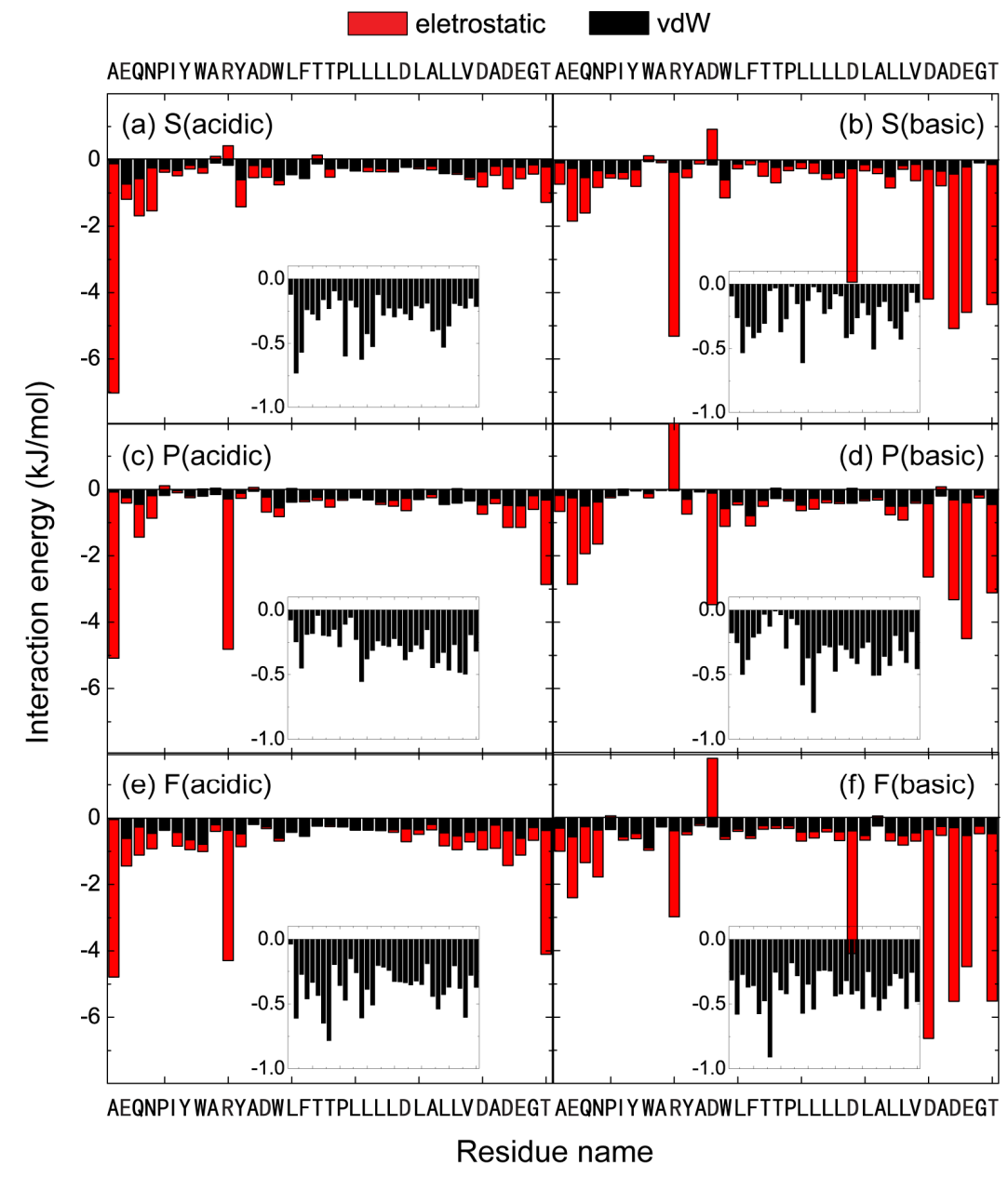


To understand further the physical driving forces underlying the $\mathrm{pH}$-dependent $\mathrm{pHLIP-POPC}$ lipid interaction, we have calculated the interaction energies of pHLIP with the polar headgroup and the hydrophobic tail group of POPC lipid (per lipid) in the six different MD runs. The results are given in Figure 7, showing that the interaction energy of the peptide-lipid headgroup is much larger than that of peptide-lipid tail group in both acidic and basic $\mathrm{pH}$ conditions, indicating that pHLIP peptide has a strong interaction with the lipid head group. Moreover, the peptide-lipid head group interaction in basic $\mathrm{pH}$ solution condition is much stronger than that in acidic $\mathrm{pH}$ condition, which may induce the peptide to bind to POPC bilayer surface at basic $\mathrm{pH}$ rather than to insert into the bilayer. The results in Figure 6, together with those in Figures 5 and 7, indicate that at acidic $\mathrm{pH}$, the protonation of the transmembrane residues Asp and the $C$-terminal residues Asp/Glu results in an increased hydrophobicity of pHLIP, facilitating their bilayer entry, while at basic $\mathrm{pH}$, the strong electrostatic interaction between Asp/Glu residues and lipid headgroups hinders bilayer insertion of the pHLIP peptide. Notably, it was suggested in a recent MD simulation study that the electrostatic attraction between transportan 10 and POPC headgroups is the main bottleneck for the peptide across the bilayer [19].

Figure 7. The interaction energy of pHLIP with POPC lipid head group (red) and tail group (black) (per lipid) in the six different MD runs: $\mathrm{S}$ (acidic), $\mathrm{S}$ (basic), $\mathrm{P}$ (acidic), $\mathrm{P}$ (basic), F (acidic), and F (basic). The data are averaged over the last $50 \mathrm{~ns}$ for each MD run.

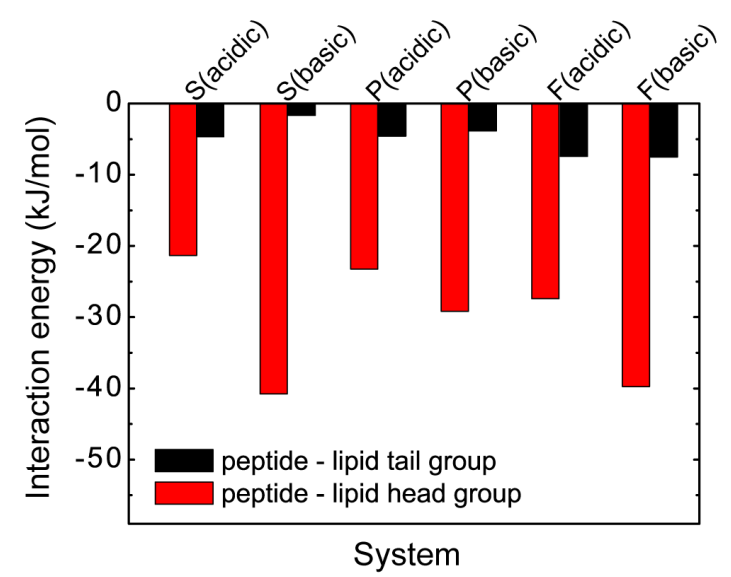

\subsection{Memebrane Insertion of pHLIP Does Not Disrupt the Integrity of POPC Bilayer Structure}

Our S (acidic) MD simulation shows that pHLIP can spontaneously insert into POPC bilayer at acidic $\mathrm{pH}$ (see Figure 1a). Recent experimental studies reported that membrane insertion of pHLIP causes minimal disturbance to POPC bilayers and does not induce membrane leakage $[1,3,6,10]$. To examine whether bilayer insertion of pHLIP at acidic $\mathrm{pH}$ would perturb the structure of POPC bialyer, we probe the area per lipid and the POPC bilayer thickness as a function of simulation time in the MD run of $\mathrm{S}$ (acidic). Figure 8a shows that the values of area per lipid, and bilayer thickness, remain almost constant during the last $50 \mathrm{~ns}$ of MD simulation. Our calculated area per lipid and bilayer thickness is $0.63 \pm 0.02 \mathrm{~nm}^{2}$ and $3.88 \pm 0.02 \mathrm{~nm}$, respectively, consistent with the experimentally measured values of $0.68 \mathrm{~nm}^{2}$ for area per lipid and $3.76 \mathrm{~nm}$ for phosphorus-phosphorus distance [32]. We also examine 
the influence of bilayer-insertion of the pHLIP on the ordering of lipid tail by calculating lipid tail order parameter $S_{\mathrm{CD}}$. Figure $8 \mathrm{~b}$ presents the average $S_{\mathrm{CD}}$ value of acyl chain 1 (sn-1) of POPC lipids over the last 50-ns MD run. For comparison, $S_{\mathrm{CD}}$ of pure POPC lipid bilayer from a 100-ns MD simulation is also given in Figure 8b. It can be seen from Figure $8 \mathrm{~b}$ that the $S_{\mathrm{CD}}$ values of POPC from MD run of $\mathrm{S}$ (acidic) overlaps well with that of the pure POPC lipid bilayer. The values of $S_{\mathrm{CD}}$, together with the calculated bilayer thickness and area per lipid, demonstrate that membrane insertion of pHLIP has negligible perturbation on the structure of POPC bilayer.

Figure 8. Characterization of the ordering of POPC bilayer. (a) Time evolution of area per lipid and bilayer thickness in simulation S (acidic); (b) Time-averaged order parameter $S_{\mathrm{CD}}$ of sn-1 chain of POPC lipids over the last 50-ns of MD run of S (acidic). For comparison, the $S_{\mathrm{CD}}$ of sn-1 chain obtained from a $20 \mathrm{~ns}$ MD run for pure POPC lipid bilayer is also presented.

(a)

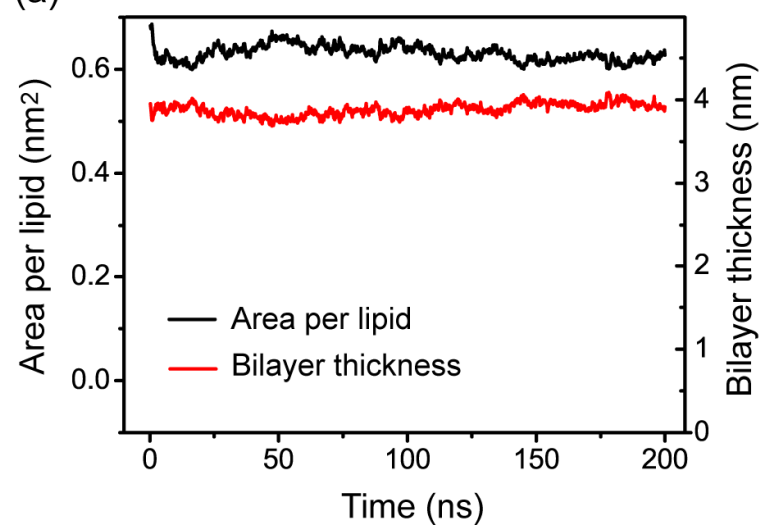

(b)

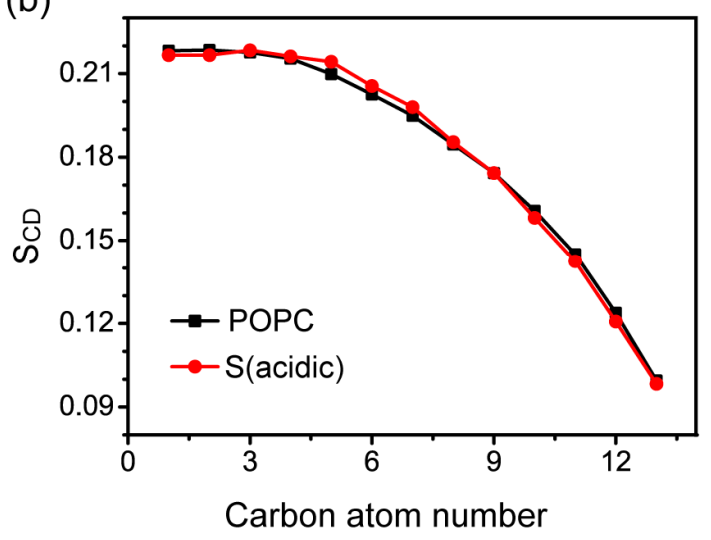

We have also examined the membrane perturbation of pHLIP in system F, where the helix spans the bilayer by calculating the area per lipid, bilayer thickness, and lipid tail order parameter $S_{\mathrm{CD}}$ of sn-1 chain. Figure S3a shows that the values of area per lipid and bilayer thickness remain almost constant during the last $50 \mathrm{~ns}$ of MD simulations, in good agreement with the results given in Figure 8a for S (acidic) system. It can be seen from Figure S3b that, although the greatest extent of bilayer perturbation is observed in $\mathrm{F}$ (acidic) system where the pHLIP spans the POPC bilayer most extensively, the $S_{\mathrm{CD}}$ value in F (acidic) system is still within the error bar of pure POPC systems, indicating that insertion of pHLIP across a POPC bilayer does not disrupt the integrity of the membrane structure.

\section{Experimental Section}

\section{1. pHLIP-POPC System}

The amino acid sequence of pHLIP used in this study is AEQNPIYWAR10 YADWLFTTPL20 LLLDLALLVD30 ADEGT. It has been reported that pHLIP is unstructured in solution and displays $\alpha$-helical structure when binding to membrane surface or inserting inside bilayer at $\mathrm{pH} 4.0$ [3]. As the coil-helix transition time for this peptide at water-bilayer interface is about $1 \mathrm{~s}$ [3], it is out of reach to capture the conformational transition from random coil to $\alpha$-helix using MD simulations at 
physiological temperatures. Moreover, recent experimental study reported that at $\mathrm{pH} 4.0$ the pHLIP forms a helix on the POPC bilayer surface, followed by bilayer insertion [3]. As a first step to understand the $\mathrm{pH}$-triggered bilayer insertion of pHLIP by conventional MD simulations, we selected the preformed helical structure as a starting state for our MD simulations. This strategy has been used by us, and other groups, for the study of bilayer insertion and membrane perturbation of antimicrobial/amyloidogenic/cell-penetrating peptides [18-20,22,24,28,33]. This helical structure is taken from the 73-107 region of transmembrane helix $\mathrm{C}$ in the NMR structure of protein Bacteriorhodopsin (pdb ID: 1R2N) [15]. The core of the pHLIP structure is an $\alpha$-helix running from residues 8-29 (corresponding to the region from residue W80 to V101 in $1 \mathrm{R} 2 \mathrm{~N}$ ).

The lipid bilayer, consists of $2 \times 64$ POPC molecules (i.e., 64 lipids in each leaflet) and the initial coordinates are obtained from a previous computational study of a pure POPC membrane [34]. The pHLIP was initially placed in three different positions with respect to the bilayer. These initial configurations are denoted as: (I) system $\mathrm{S}$, in which pHLIP was initially placed in aqueous solution with its backbone parallel to the POPC bilayer surface and the center-of-mass (COM) of the pHLIP backbone is $1.8 \mathrm{~nm}$ away from the membrane surface (see the snapshot at $t=0 \mathrm{~ns}$ in Figure 1a,b); (II) system P, where pHLIP was partially-inserted in POPC bilayer with the $C$-terminal residues 24-35 embedded inside lipid bilayer (see the snapshot at $t=0 \mathrm{~ns}$ in Figure 2a,b); (III) system F, where pHLIP was fully-inserted in POPC bilayer with the pHLIP helix spanning the bilayer (see the snapshot at $t=0 \mathrm{~ns}$ in Figure 4a,b). All pHLIP-bilayer systems are fully solvated in water. Counterions $\left(\mathrm{Cl}^{-}\right.$or $\left.\mathrm{Na}^{+}\right)$ are added to neutralize the system. The VMD software was used to display the systems [35].

\subsection{Molecular Dynamics Simulations}

All MD simulations have been performed in the isothermal-isobaric (NPT) ensemble using the GROMACS 3.3 software package [36]. The Gromos 87 force field [37] is used to describe the peptide and bilayer interactions. A water molecule is modeled by the simple point charge (SPC) model [38]. Bond length of the peptides and lipids are constrained with LINCS [39] and water geometries are constrained with SETTLE [40]. This allows an integration time of 2 fs. Long-range electrostatic interactions are calculated using the Particle Mesh Ewald (PME) [41] method, as recommended for membrane simulations $[42,43]$. Van der Waals $(v d W)$ interactions are calculated using a cutoff of $1.4 \mathrm{~nm}$ [42]. The lipid bilayer, water, peptide, and ions are weakly coupled (with a coupling constant of $0.1 \mathrm{ps)}$ to temperature bath [44] separately at $T=315 \mathrm{~K}$, above the gel-liquid crystal phase transition temperature $(270 \mathrm{~K})$ of POPC lipid bilayer [45]. The pressure is also weakly coupled (with a coupling constant of $1.0 \mathrm{ps}$, and compressibility of $4.5 \times 105$ bar) to 1 bar using semi-isotropic coupling scheme, in which the lateral (parallel to bilayer surface) and perpendicular (along bilayer normal) pressure are coupled independently [42]. A 200-ns MD simulation has been carried out for each system ( $\mathrm{S}, \mathrm{P}, \mathrm{F}$ system) in both acidic and basic $\mathrm{pH}$ environment and there are six MD runs in total. The six MD runs are labeled as S (acidic), S (basic), P (acidic), P (basic), F (acidic), and F (basic). Here the letters S, P, F represent surface, partial-insertion, and full-insertion system, respectively. All MD simulations have been performed using periodic boundary condition in a rectangular box. A summary of the MD setup details is given in Table 1. 
Table 1. Summary for the setup details of the six 200-ns MD runs at $315 \mathrm{~K}$ for three different systems S, P, and F. For each system, we describe the name of the system, the initial membrane insertion state of pHLIP, the name of MD runs, the initial state of each MD run, the total number of atoms, the size of simulation box and the length of each MD run.

\begin{tabular}{ccccccc}
\hline System & $\begin{array}{c}\text { Insertion state of } \\
\text { pHLIP }\end{array}$ & $\begin{array}{c}\text { Name of } \\
\text { MD run }\end{array}$ & $\begin{array}{c}\text { Initial } \\
\text { state }\end{array}$ & $\begin{array}{c}\text { Number } \\
\text { of atoms }\end{array}$ & $\begin{array}{c}\text { Simulation box } \\
\text { size (nm }\end{array}$ & $\begin{array}{c}\text { Length of } \\
\text { MD run (ns) }\end{array}$ \\
\hline \multirow{2}{*}{ S } & Surface & S (acidic) & Figure 1a & 27,083 & $6.6 \times 6.5 \times 9.0$ & 200 \\
& Surface & S (basic) & Figure 1b & 27,066 & $6.6 \times 6.5 \times 9.0$ & 200 \\
\hline \multirow{2}{*}{ P } & Partial insertion & P (acidic) & Figure 2a & 24,347 & $6.5 \times 6.5 \times 9.0$ & 200 \\
& Partial insertion & P (basic) & Figure 2b & 24,345 & $6.5 \times 6.5 \times 9.0$ & 200 \\
\hline \multirow{2}{*}{ F } & Full insertion & F (acidic) & Figure 4a & 16,808 & $6.4 \times 6.3 \times 6.5$ & 200 \\
& Full insertion & F (basic) & Figure 4b & 16,789 & $6.4 \times 6.3 \times 6.5$ & 200 \\
\hline
\end{tabular}

\subsection{Analysis}

All of the analyses have been done using our in-house developed codes and the analysis tools available in the GROMACS 3.3 package [36]. The ordering of POPC lipid bilayer is characterized by lipid tail order parameter $\left(\mathrm{S}_{\mathrm{CD}}\right)$, bilayer thickness, and the area per lipid. The bilayer thickness is estimated by the average of the phosphorus-phosphorus distance. The Lipid tail order parameter $\mathrm{S}_{\mathrm{CD}}$ is calculated using the formula: $S_{\mathrm{CD}}=0.5 \times\left\langle 3 \cos ^{2} \theta-1\right\rangle$, where, $\theta$ is the angle between the $\mathrm{C}-\mathrm{H}$ bond vector (in the simulation) or the C-D bond vector (in the experiment) and the bilayer normal. The angular brackets imply an average over time and lipid molecules [46,47]. The secondary structure analysis is performed using DSSP program [48]. The insertion depth of pHLIP peptide in POPC lipid bilayer is estimated by the $z$-position of the COM of peptide backbone and the $z$-position of the most deeply inserted atom of residue Leu28 in the bilayer. The $z$-position is averaged over the last $50 \mathrm{~ns}$ for each MD run. The interaction energy of each amino acid residue with the POPG lipid bilayer (per lipid), and the interaction energy of pHLIP with POPC lipid head group and tail group (per lipid) in the six different MD runs are also calculated, averaged over the last $50 \mathrm{~ns}$ of each MD run.

\section{Conclusions}

In summary, we have investigated the detailed interactions of pHLIP with POPC bilayers at acidic and basic $\mathrm{pH}$ conditions by performing six 200-ns all-atom MD simulations starting from three different configurations. When the peptide was placed in aqueous solution parallel to the bilayer surface, pHLIP is quickly adsorbed to the bilayer surface and can spontaneously insert in POPC bilayer at acidic $\mathrm{pH}$, while it binds to the membrane surface without inserting at basic $\mathrm{pH}$. Calculation of lipid order parameters demonstrates that bilayer insertion of pHLIP does not disrupt the structure of POPC bilayer, consistent with recent experimental studies showing that membrane insertion of pHLIP causes minimal disturbance to POPC bilayers and does not induce membrane leakage $[1,6,10]$. When the peptide was partially inserted in POPC bilayer, it can insert deep into the bilayer at acidic $\mathrm{pH}$ while moves towards the bilayer surface at basic $\mathrm{pH}$. When it was fully inserted inside POPC bilayer, pHLIP keeps intact $\alpha$-helical structure at acidic $\mathrm{pH}$ while the helix unfolds at basic $\mathrm{pH}$. The pHLIP at acidic 
$\mathrm{pH}$ is more hydrophobic, energetically more favorable to stay inside the hydrophobic tail region of POPC bilayer At acidic $\mathrm{pH}$, the protonation of transmembrane residues Asp and the $C$-terminal residues Asp/Glu facilitates their bilayer insertion due to the increased hydrophobicity of pHLIP, while at basic $\mathrm{pH}$, the strong electrostatic interaction between Asp/Glu residues and lipid headgroups hinders the bilayer insertion. The total potential energy analysis indicates that it is energetically more favorable for the pHLIP to stay inside the membrane as an $\alpha$-helical structure at acidic $\mathrm{pH}$ while bind to the membrane surface at basic $\mathrm{pH}$. This study provides atomic details for the $\mathrm{pH}$-dependent $\mathrm{pHLIP-POPC}$ interaction and improves our understanding toward the molecular mechanism underlying the pH-triggered bilayer insertion of pHLIP.

\section{Acknowledgments}

This work was supported by the National Natural Science Foundation of China (11074047) and Research Fund for the Doctoral Program of Higher Education of China (RFDP-20100071110006). Simulations were performed the National High Performance Computing Center of Fudan University.

\section{Conflict of Interest}

The authors declare no conflict of interest.

\section{References}

1. Reshetnyak, Y.K.; Andreev, O.A.; Lehnert, U.; Engelman, D.M. Translocation of molecules into cells by ph-dependent insertion of a transmembrane helix. Proc. Natl. Acad. Sci. USA 2006, 103, 6460-6465.

2. An, M.; Wijesinghe, D.; Andreev, O.A.; Reshetnyak, Y.K.; Engelman, D.M. $\mathrm{Ph}$-(low)-insertion-peptide (phlip) translocation of membrane impermeable phalloidin toxin inhibits cancer cell proliferation. Proc. Natl. Acad. Sci. USA 2010, 107, 20246-20250.

3. Andreev, O.A.; Karabadzhak, A.G.; Weerakkody, D.; Andreev, G.O.; Engelman, D.M.; Reshetnyak, Y.K. Ph (low) insertion peptide (phlip) inserts across a lipid bilayer as a helix and exits by a different path. Proc. Natl. Acad. Sci. USA 2010, 107, 4081-4086.

4. Reshetnyak, Y.K.; Andreev, O.A.; Segala, M.; Markin, V.S.; Engelman, D.M. Energetics of peptide (phlip) binding to and folding across a lipid bilayer membrane. Proc. Natl. Acad. Sci. USA 2008, 105, 15340-15345.

5. Hunt, J.F.; Rath, P.; Rothschild, K.J.; Engelman, D.M. Spontaneous, ph-dependent membrane insertion of a transbilayer alpha-helix. Biochemistry 1997, 36, 15177-15192.

6. Reshetnyak, Y.K.; Segala, M.; Andreev, O.A.; Engelman, D.M. A monomeric membrane peptide that lives in three worlds: In solution, attached to, and inserted across lipid bilayers. Biophys. J. 2007, 93, 2363-2372.

7. Andreev, O.A.; Dupuy, A.D.; Segala, M.; Sandugu, S.; Serra, D.A.; Chichester, C.O.; Engelman, D.M.; Reshetnyak, Y.K. Mechanism and uses of a membrane peptide that targets tumors and other acidic tissues in vivo. Proc. Natl. Acad. Sci. USA 2007, 104, 7893-7898. 
8. Musial-Siwek, M.; Karabadzhak, A.; Andreev, O.A.; Reshetnyak, Y.K.; Engelman, D.M. Tuning the insertion properties of phlip. Biochim. Biophys. Acta 2010, 1798, 1041-1046.

9. Makovitzki, A.; Fink, A.; Shai, Y. Suppression of human solid tumor growth in mice by intratumor and systemic inoculation of histidine-rich and ph-dependent host defense-like lytic peptides. Cancer Res. 2009, 69, 3458-3463.

10. Zoonens, M.; Reshetnyak, Y.K.; Engelman, D.M. Bilayer interactions of phlip, a peptide that can deliver drugs and target tumors. Biophys. J. 2008, 95, 225-235.

11. Thevenin, D.; An, M.; Engelman, D.M. Phlip-mediated translocation of membrane-impermeable molecules into cells. Chem. Biol. 2009, 16, 754-762.

12. Vavere, A.L.; Biddlecombe, G.B.; Spees, W.M.; Garbow, J.R.; Wijesinghe, D.; Andreev, O.A.; Engelman, D.M.; Reshetnyak, Y.K.; Lewis, J.S. A novel technology for the imaging of acidic prostate tumors by positron emission tomography. Cancer Res. 2009, 69, 4510-4516.

13. Andreev, O.A.; Engelman, D.M.; Reshetnyak, Y.K. Targeting acidic diseased tissue: New technology based on use of the ph (low) insertion peptide (phlip). Chim. Oggi. 2009, 27, 34-37.

14. Tang, J.; Gai, F. Dissecting the membrane binding and insertion kinetics of a phlip peptide. Biochemistry 2008, 47, 8250-8252.

15. Patzelt, H.; Simon, B.; terLaak, A.; Kessler, B.; Kuhne, R.; Schmieder, P.; Oesterhelt, D.; Oschkinat, $H$. The structures of the active center in dark-adapted bacteriorhodopsin by solution-state nmr spectroscopy. Proc. Natl. Acad. Sci. USA 2002, 99, 9765-9770.

16. Gkeka, P.; Sarkisov, L. Interactions of phospholipid bilayers with several classes of amphiphilic alpha-helical peptides: Insights from coarse-grained molecular dynamics simulations. J. Phys. Chem. B 2010, 114, 826-839.

17. Rychkova, A.; Vicatos, S.; Warshel, A. On the energetics of translocon-assisted insertion of charged transmembrane helices into membranes. Proc. Natl. Acad. Sci. USA 2010, 107, 17598-17603.

18. Soliman, W.; Bhattacharjee, S.; Kaur, K. Interaction of an antimicrobial peptide with a model lipid bilayer using molecular dynamics simulation. Langmuir 2009, 25, 6591-6595.

19. Dunkin, C.M.; Pokorny, A.; Almeida, P.F.; Lee, H.S. Molecular dynamics studies of transportan 10 (tp10) interacting with a popc lipid bilayer. J. Phys. Chem. B 2011, 115, 1188-1198.

20. Chang, Z.; Luo, Y.; Zhang, Y.; Wei, G. Interactions of abeta25-35 beta-barrel-like oligomers with anionic lipid bilayer and resulting membrane leakage: An all-atom molecular dynamics study. J. Phys. Chem. B 2011, 115, 1165-1174.

21. Yesylevskyy, S.; Marrink, S.J.; Mark, A.E. Alternative mechanisms for the interaction of the cell-penetrating peptides penetratin and the tat peptide with lipid bilayers. Biophys. J. 2009, 97, 40-49.

22. Fleming, E.; Maharaj, N.P.; Chen, J.L.; Nelson, R.B.; Elmore, D.E. Effect of lipid composition on buforin ii structure and membrane entry. Proteins 2008, 73, 480-491.

23. Ulmschneider, M.B.; Doux, J.P.; Killian, J.A.; Smith, J.C.; Ulmschneider, J.P. Mechanism and kinetics of peptide partitioning into membranes from all-atom simulations of thermostable peptides. J. Am. Chem. Soc. 2010, 132, 3452-3460.

24. Elmore, D.E. Insights into buforin ii membrane translocation from molecular dynamics simulations. Peptides 2012, 38, 357-362. 
25. Tieleman, D.P.; Sansom, M.S.; Berendsen, H.J. Alamethicin helices in a bilayer and in solution: Molecular dynamics simulations. Biophys. J. 1999, 76, 40-49.

26. Leontiadou, H.; Mark, A.E.; Marrink, S.J. Antimicrobial peptides in action. J. Am. Chem. Soc. 2006, 128, 12156-12161.

27. Jang, H.; Ma, B.; Woolf, T.B.; Nussinov, R. Interaction of protegrin-1 with lipid bilayers: Membrane thinning effect. Biophys. J. 2006, 91, 2848-2859.

28. Zhang, Y.; Luo, Y.; Deng, Y.; Mu, Y.; Wei, G. Lipid interaction and membrane perturbation of human islet amyloid polypeptide monomer and dimer by molecular dynamics simulations. PLoS One 2012, 7, e38191.

29. Yu, X.; Zheng, J. Cholesterol promotes the interaction of alzheimer beta-amyloid monomer with lipid bilayer. J. Mol. Biol. 2012, 421, 561-571.

30. Tofoleanu, F.; Buchete, N.V. Molecular interactions of alzheimer's abeta protofilaments with lipid membranes. J. Mol. Biol. 2012, 421, 572-586.

31. Jia, Y.; Qian, Z.; Zhang, Y.; Wei, G. Adsorption and orientation of human islet amyloid polypeptide (hiapp) monomer at anionic lipid bilayers: Implications for membrane-mediated aggregation. Int. J. Mol. Sci. 2013, 14, 6241-6258.

32. Kučerka, N.; Tristram-Nagle, S.; Nagle, J. Structure of fully hydrated fluid phase lipid bilayers with monounsaturated chains. J. Membr. Biol. 2006, 208, 193-202.

33. Duan, M.; Fan, J.; Huo, S. Conformations of islet amyloid polypeptide monomers in a membrane environment: Implications for fibril formation. PLoS One 2012, 7, e47150.

34. Hoff, B.; Strandberg, E.; Ulrich, A.S.; Tieleman, D.P.; Posten, C. 2h-nmr study and molecular dynamics simulation of the location, alignment, and mobility of pyrene in popc bilayers. Biophys. J. 2005, 88, 1818-1827.

35. Humphrey, W.; Dalke, A.; Schulten, K. Vmd: Visual molecular dynamics. J. Mol. Graph. 1996, 14, 33-38.

36. Van Der Spoel, D.; Lindahl, E.; Hess, B.; Groenhof, G.; Mark, A.E.; Berendsen, H.J.C. Gromacs: Fast, flexible, and free. J. Comput. Chem. 2005, 26, 1701-1718.

37. Piccinini, E.; Ceccarelli, M.; Affinito, F.; Brunetti, R.; Jacoboni, C. Biased molecular simulations for free-energy mapping: A comparison on the kcsa channel as a test case. J. Chem. Theory Comput. 2007, 4, 173-183.

38. Berendsen, H.J.C.; Postma, J.P.M.; Gunsteren, W.F.V.; Hermans, J. Interaction Models for Water in Relation to Protein Hydration; D. Reidel Pulishing Co.: Dordrecht, The Netherlands, 1981; pp. 331-342.

39. Hess, B.; Bekker, H.; Berendsen, H.J.C.; Fraaije, J.G.E.M. Lincs: A linear constraint solver for molecular simulations. J. Comput. Chem. 1997, 18, 1463-1472.

40. Miyamoto, S.; Kollman, P.A. Settle: An analytical version of the shake and rattle algorithm for rigid water models. J. Comput. Chem. 1992, 13, 952-962.

41. Darden, T.; York, D.; Pedersen, L. Particle mesh ewald: An $n \cdot \log (n)$ method for ewald sums in large systems. J. Chem. Phys. 1993, 98, 10089-10092.

42. Kandt, C.; Ash, W.L.; Tieleman, D.P. Setting up and running molecular dynamics simulations of membrane proteins. Methods 2007, 41, 475-488. 
43. Patra, M.; Karttunen, M.; Hyvonen, M.T.; Falck, E.; Lindqvist, P.; Vattulainen, I. Molecular dynamics simulations of lipid bilayers: Major artifacts due to truncating electrostatic interactions. Biophys. J. 2003, 84, 3636-3645.

44. Vermeer, L.S.; de Groot, B.L.; Reat, V.; Milon, A.; Czaplicki, J. Acyl chain order parameter profiles in phospholipid bilayers: Computation from molecular dynamics simulations and comparison with 2h nmr experiments. Eur. Biophys. J. 2007, 36, 919-931.

45. Seelig, J.; Waespe-Sarcevic, N. Molecular order in cis and trans unsaturated phospholipid bilayers. Biochemistry 1978, 17, 3310-3315.

46. Chau, P.L.; Hardwick, A.J. A new order parameter for tetrahedral configurations. Mol. Phys. 1998, 93, 511-518.

47. Petrache, H.I.; Tu, K.; Nagle, J.F. Analysis of simulated nmr order parameters for lipid bilayer structure determination. Biophys. J. 1999, 76, 2479-2487.

48. Kabsch, W.; Sander, C. Dictionary of protein secondary structure: Pattern recognition of hydrogen-bonded and geometrical features. Biopolymers 1983, 22, 2577-2637.

(C) 2013 by the authors; licensee MDPI, Basel, Switzerland. This article is an open access article distributed under the terms and conditions of the Creative Commons Attribution license (http://creativecommons.org/licenses/by/3.0/). 